\title{
Comparison of Edge Detection Techniques for Iris Recognition
}

\author{
Satbir Kaur \\ Research Scholar \\ CSE \& IT Department \\ Baba Banda Singh Bahadur \\ Engineering College, Fatehgarh Sahib
}

\author{
Ishpreet Singh \\ Assistant Professor \\ CSE \& IT Department \\ Baba Banda Singh Bahadur \\ Engineering College, Fatehgarh Sahib
}

\begin{abstract}
Nowadays security and authentication are the foremost parts of our daily life. Iris is the one of the most reliable organ or part of the human body which can be used for identification and authentication purpose. This paper examines for edge detection techniques use for iris recognition system .Between the prewitt,sobel,LoG,Min.contructor of laplacian edge detector techniques the experimental results show that minimum constructor of laplacian edge detector(Hybrid) has better ability to detect edges in digital image.
\end{abstract}

\section{General Terms}

Your general terms must be any term which can be used for general classification of the submitted material such as Pattern Recognition, Security, Algorithms et. al.

\section{Keywords}

Sobel, Prewitt, LoG, Min.constructor of laplacian edge detector(Hybrid), SSIM

\section{INTRODUCTION}

The term "biometric" is formed by joining two words "bio" which means life and "metric" which means measure. By the term "biometric" means measurement and statiscal analysis of characteristics of people which are either physical or behavioural. Biometric is the study of distinguishable biological characteristics that are unique and measureable like iris, face, colour of eye, etc. They can be used for the purpose of verification and now a days it is very popular and safe.A person can be more secure using biometric as it ascertain whether a person is who he claimed to be or not. The main aim of "iris recognizaton" is used to verify and authenticate a person from his or her unique pattern of iris. The patterns of iris are different and unique for each individual. The unique pattern of iris is shown in the following figure:

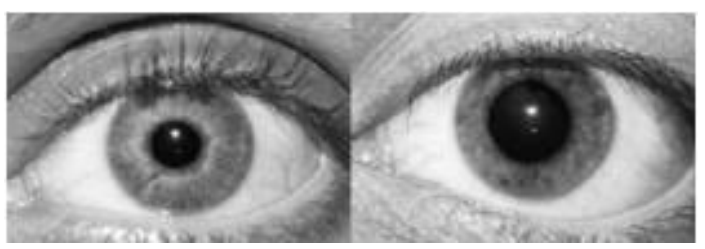

Fig 1.1: Distinctiveness of human iris

Iris Recognization system has mainly eye capturing, image pre-processing, edge detection,feature extraction and pattern matching.From alll of the above edge detection plays an impertant role in iris recognization system. . Edge detection techniques transform images into edge images benefiting from the changes of grey tones in the images [27]. Detection of edges for an image may help for image segmentation, data compression, and also help for well matching, such as image reconstruction and so on [28]. Various types of edge detection techniques are used and available for image extraction etc. Each technique is constructed to be sensible for assertive types of edges. Among them Prewitt,Sobel,Robert ,Canny,Laplacian and Laplacian of Gaussian are foremost techniques.

\section{METHODOLOGY}

Edge detection is a type of image segmentation techniques which determines the presence of an edge or line in an image and outlines them in an appropriate way [9]. The aim of edge detection is to reduce the image data so that lesser amount of data is being refined.Generally, an edge is defined as the boundary pixels that connect two seprate regions with changing image amplitude characteristics.The methodology used in this paper as follows:

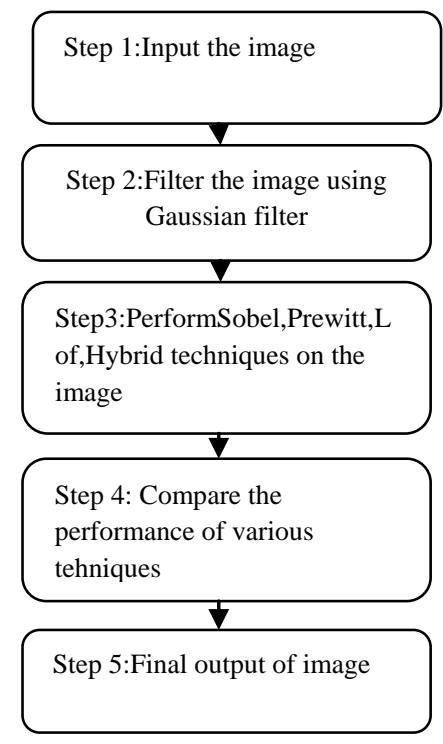

Fig 2.1: Methology used in the paper

\subsection{Gaussian Filtering}

Gaussian filtering has been intensively studied in image processing and computer vision. Using Gaussian filter for noise suppression, the noise is smoothed out, at the same time the signal is also distorted. The use of Gaussian filter as preprocessing for edge detection will also give rise to edge position displacement, edges vanishing, and phantom edges.

\subsection{Sobel Operator}

It is $3 \times 3$ convolution kernels. One kernel is simply the other rotated by $90^{\circ}$. It is a row edge detector. 

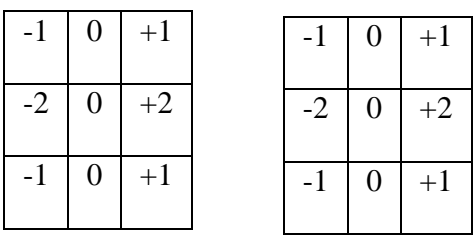

Gx

Gy

Fig 2.2: Sobel mask filter in $\mathrm{X}$ direction and $\mathrm{Y}$ direction

GX and GY are the common masks used in Sobel Operator. This figure shows the masks used by Sobel operator. The kernel can be applied separately to input image for obtaining gradient component in each orientation i.e. GX and GY. The magnitude is:

$|\mathrm{G}|=\sqrt{ } \mathrm{GX} 2+\mathrm{GY} 2$

And its approximation is:

$|\mathrm{G}|=|\mathrm{GX}|+|\mathrm{GY}|$

The orientation of angle is:

$\theta=\arctan (\mathrm{GX} / \mathrm{GY})$

\subsection{Prewitt Operator:}

It is similar [11] to the Sobel Operator and is used to detect vertical and horizontal edges in an image. The prewitt mask operator used is as follows:

\begin{tabular}{|l|l|l|}
\hline-1 & 0 & 1 \\
\hline-1 & 0 & 1 \\
\hline-1 & 0 & 1 \\
\hline
\end{tabular}

Px

\begin{tabular}{|l|l|l|}
\hline 1 & 1 & 1 \\
\hline 0 & 0 & 0 \\
\hline-1 & -1 & -1 \\
\hline
\end{tabular}

Py
Fig 2.3: Prewitt mask filter in $\mathrm{X}$ direction and $\mathrm{Y}$ direction

\subsection{Laplacian of Gaussian (LoG):}

It was proposed by Marr (1982). The LoG of an image $f(x, y)$ is a second derivative defined as

$$
\nabla_{2 \mathrm{f}=\frac{\partial^{2} f}{\partial x^{2}}}+\frac{\partial^{2} f}{\partial y^{2}}
$$

It first smoothes the image and then computes the Laplacian. This yields in double edge image; hence for finding the edge the zero crossing between the double edges is taken.

The Laplacian of an image with the pixel intensity value $\mathrm{L}(\mathrm{x}, \mathrm{y})$ is given by:

$$
L(x, y)=\frac{\partial^{2} I}{\partial x^{2}}+\frac{\partial^{2} I}{\partial y^{2}}
$$

The commonly used discrete approximations to Laplacian filter are:

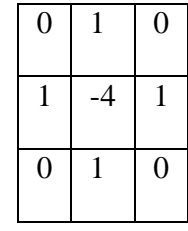

Lx

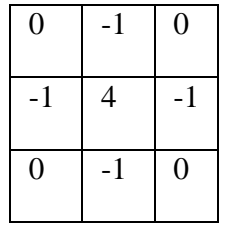

Ly
Fig 2.4: Laplacian filter in $\mathrm{X}$ direction and $\mathrm{Y}$ direction

\subsection{Min Constructor With Laplacian Edge Detector}

Min Construction Method :

The lower constructor is a generalization of the tn-processing A t-norm $\mathrm{T}:[0,1] 2 \rightarrow[0,1]$ is an associative, commutative, increasing function, such that, $\mathrm{T}(1, \mathrm{x})=\mathrm{x}$ for all $\mathrm{x} \in[0,1]$. At-norm $\mathrm{T}$ is called idempotent if $\mathrm{T}(\mathrm{x}, \mathrm{x})=\mathrm{x}$ for all $\mathrm{x} \in[0,1]$. The four basic t-norms are as follows

1. The minimum $\mathrm{TM}(\mathrm{x}, \mathrm{y})=\min (\mathrm{x}, \mathrm{y})$.

2. The product $\mathrm{TP}(\mathrm{x}, \mathrm{y})=\mathrm{x} \cdot \mathrm{y}$.

3. The Łukasiewicz t-norm

4. $\operatorname{TL}(\mathrm{x}, \mathrm{y})=\max (\mathrm{x}+\mathrm{y}-1,0)$.

5. The nilpotent minimum t-norm

6. $\operatorname{TnM}(x, y)=\min (x, y)$, if $x+y>1$

0 , otherwise.

- Let $\mathrm{R} \in \mathrm{F}(\mathrm{X} \times \mathrm{Y})$ be an FR. Consider two t-norms $\mathrm{T} 1$ and $\mathrm{T} 2$ and two values $\mathrm{n}, \mathrm{m} \in \mathrm{N}$ so that $\mathrm{n} \leq \mathrm{P}$ $-1 / 2$, and $\mathrm{m} \leq \mathrm{Q}-1 / 2$. We define the lower constructor associated with $\mathrm{T} 1, \mathrm{~T} 2, \mathrm{n}$, and $\mathrm{m}$ in the following way:

- $\quad \mathrm{L} n, \mathrm{mT} 1, \mathrm{~T} 2: \mathrm{F}(\mathrm{X} \times \mathrm{Y}) \rightarrow$ given by

$\mathrm{L} \mathrm{n}, \mathrm{mT} 1, \mathrm{~T} 2[\mathrm{R}](\mathrm{x}, \mathrm{y})=\mathrm{T} 1 \mathrm{~m}, \mathrm{n}(\mathrm{T} 2(\mathrm{R}(\mathrm{x}-\mathrm{i}, \mathrm{y}-\mathrm{j}), \mathrm{R}(\mathrm{x}, \mathrm{y}$

$$
\mathrm{J}=-\mathrm{m} \text { for } \text { all }(\mathrm{x}, \mathrm{y}) \in(\mathrm{X}, \mathrm{Y})
$$

The Algorithm begins with reading an $\mathrm{M} x \mathrm{~N}$ image. The first set of nine pixels of a $3 \times 3$ window are chosen with central pixel having values $(2,2)$ i.e for each pixel(i,j) we are taking the 8 neighbourhood of $(i, j)$.After the initialization, the pixel values are initially marked as edge pixel after an observation to the 8 neighbourhood. After the subjection of the pixel values the algorithm generates an intermediate image using a construction method stated below. It is checked whether all pixels have been checked or now, if not then first the horizontal coordinate pixels are checked. If all horizontal pixels have been checked the vertical pixels are checked else the horizontal pixel is incremented to retrieve the next set of pixels of a window. In this manner the window shifts and checks all the pixels in one horizontal line then increments to check the next vertical location. 


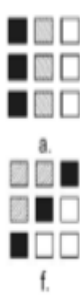

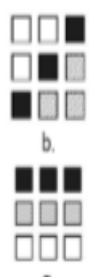

g.

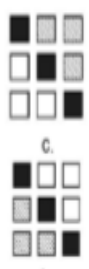

h

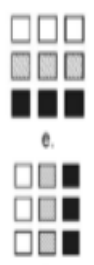

i.

Fig 2.5:Initial conditions to check 8 neighbourhood
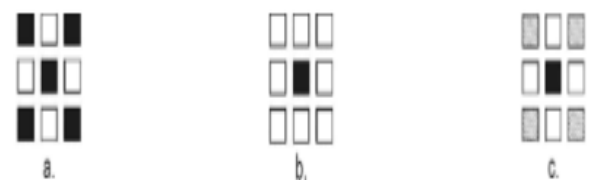

Fig2.6: (a,b)Type of unwanted edge pixels (c)condition for removal of unwanted edge pixels

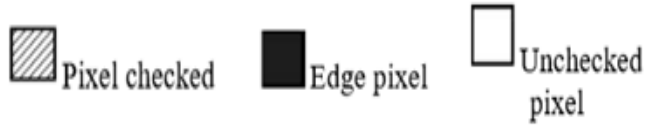

After edge highlighting image is subjected to another set of condition with the help of which the unwanted parts of the output image of type shown in Fig.(a-b) are removed to generate an image which contains only the edges associated with the input image

Phase 1:

- Input: An image $\mathrm{P}$ of $\mathrm{Mx} \mathrm{N}$ pixels

- $\quad$ Output: An image Q of M x N pixels

Initial Edge Detection (A, B) using Min Construction

For $\mathrm{I} \leftarrow 2$ to $\mathrm{M}-1$

For $\mathrm{J} \leftarrow 2$ to $\mathrm{N}-1$

If $\mathrm{P}(\mathrm{I}-1, \mathrm{~J})>\mathrm{P}(\mathrm{I}-1, \mathrm{~J}+1)$

Then If $\mathrm{P}(\mathrm{I}-1, \mathrm{~J}-1)>\mathrm{P}(\mathrm{I}, \mathrm{J})$

Then If $\mathrm{P}(\mathrm{I}, \mathrm{J}-1)>\mathrm{P}(\mathrm{I}+1, \mathrm{~J}-1)$

Then

$\mathrm{Q}(\mathrm{I}-1, \mathrm{~J}+1) \leftarrow 0$

$\mathrm{Q}(\mathrm{I}, \mathrm{J}) \leftarrow 0$

$\mathrm{Q}(\mathrm{I}+1, \mathrm{~J}-1) \leftarrow 0$

End For

End For

For $\mathrm{I} \leftarrow 2$ to $\mathrm{M}-1$

For $\mathrm{J} \leftarrow 2$ to $\mathrm{N}-1$

If $\mathrm{Q}(\mathrm{I}-1, \mathrm{~J})=255 \& \mathrm{Q}(\mathrm{I}, \mathrm{J})=0 \& \mathrm{Q}(\mathrm{I}+1, \mathrm{~J})=255 \& \mathrm{Q}(\mathrm{I}, \mathrm{J}-1)=255$

Then $\mathrm{Q}(\mathrm{I}, \mathrm{J})$ is minimum and highlighted as edge initially.

End For

In the above algorithm Min construction[25] is used but not as after fuzzification the membership values would become fractions that can't be stored in unsigned char. Hence the same technique of min construction is used but on true picture and taking into consideration 8-nbd of a pixel $(i, j)$. We can observe in the above algorithm written for a particular fuzzy condition that the nesting of statements is done in a manner that only the edge associated pixels are granted black pixel values and initially min valued edge pixels are given white value. These pixels are initially marked as edge.

Phase 2.

- Input: An image Q (256 color true bmp image) of size $\mathrm{MxN}$

- Output: Edge image of size $\mathrm{MxN}$

We now use Laplacian of Gaussian (log) operator[26] on the intermediate image to get the edge image. And In this way whatever image is being constructed is compared with edges found on same image by other existing techniques. The phase 1 actually performs a check like

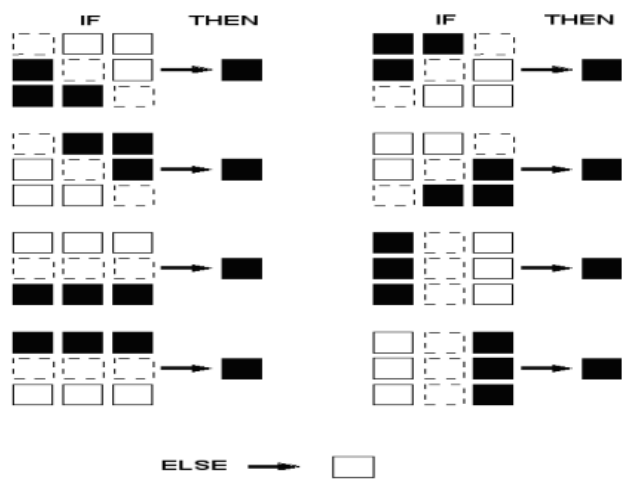

Fig2.7: Black pixels are having min values

\section{EXPERIMENTAL RESULTS}

The image of iris is considered as the input for applying the edge detection techniques. Here first iris image is filterd by using Gaussian filtering so that noise can be removed and then edge detection is used to extract the edge points in the characters in the iris images. Four Edge detection techniques have been analyzed and compared to detect the edgesin the iris image. Edges of an image are detected using Sobel, Prewitt, Laplacian of Gaussian (LoG) and minimum constructor with lalacian edge detectors(Hybrid). From the experimental results, the performance of Hybrid provides better result than other edge detection techniques for iris image .This work has been calculated by using SSIM (Structural Similarity Index for Measuring), which is used for measuring the similarity between two images. SSIM designed to improve on traditional methods such as peak signal-tonoise ratio (PSNR) and mean squared error (MSE). The improved SSIM measure can be used for edge detection (Parameterization), modulating of edge tracing outputs and comparison of edge tracing for real and fake images.

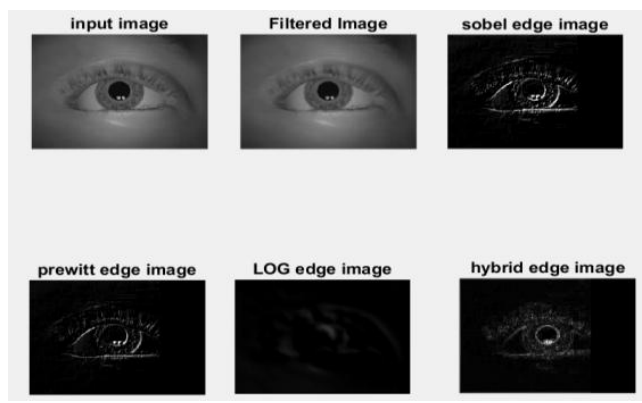

Fig3.1: output of various edge detection techniques applied in (a) image 


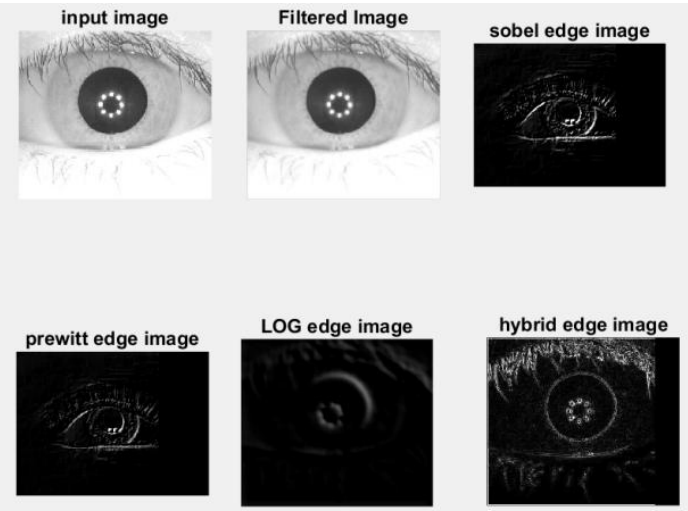

Fig3.2: output of various edge detection techniques applied in (b) image

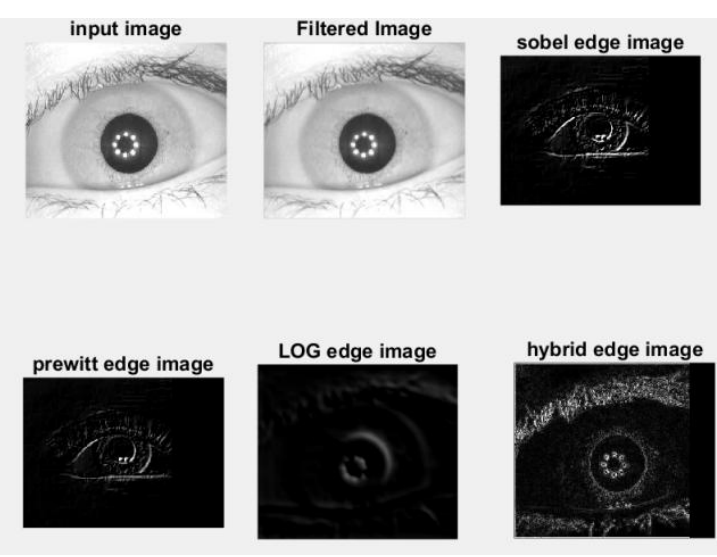

Fig3.3: output of various edge detection techniques applied in (c) image

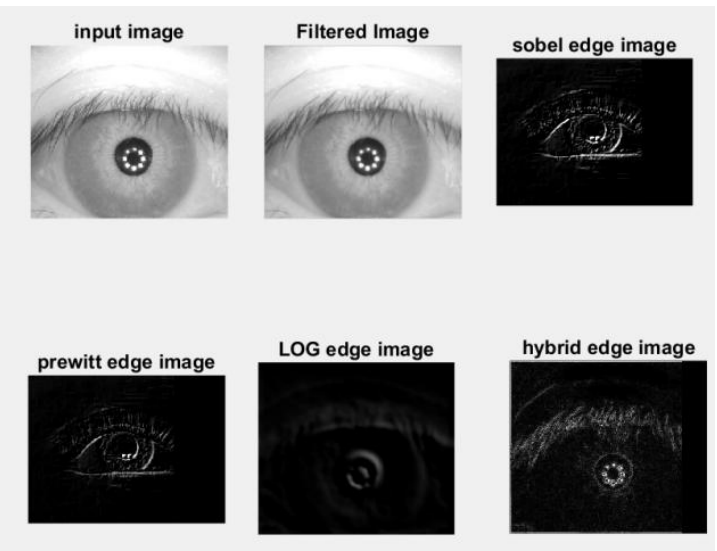

Fig3.4: output of various edge detection techniques applied in (d) image

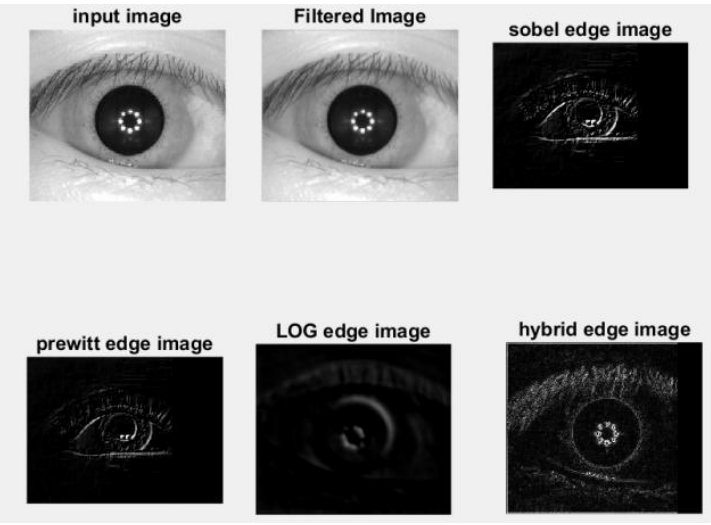

Fig3.5: output of various edge detection techniques applied in (e) image

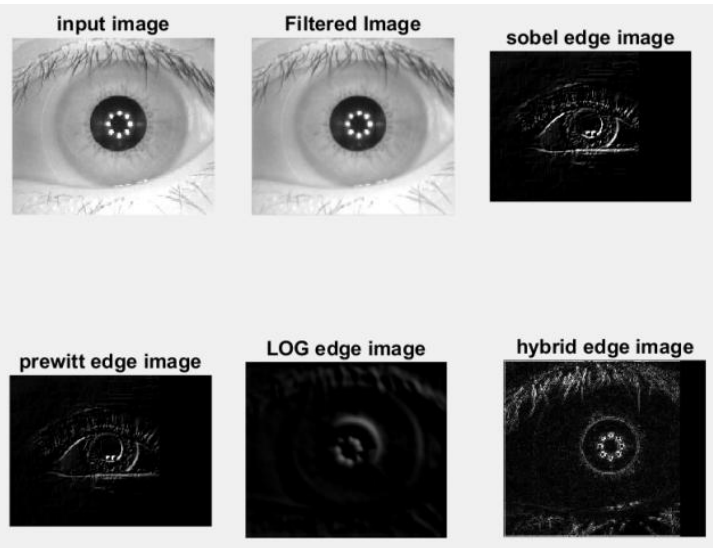

Fig3.6: output of various edge detection techniques applied in (f) image

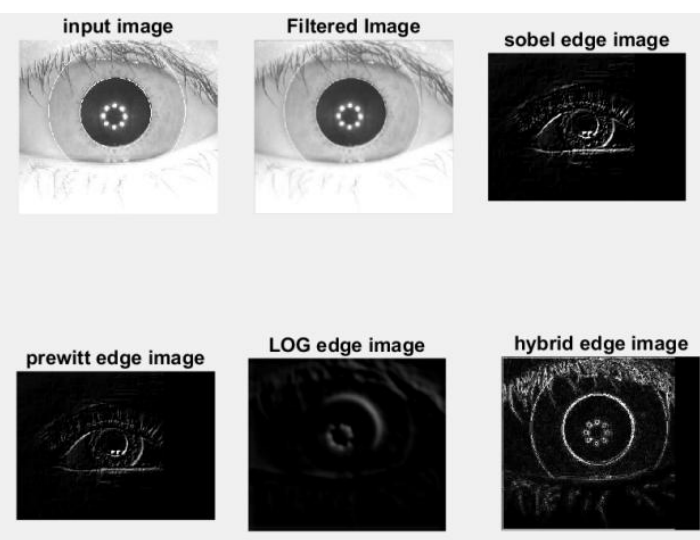

Fig3.7: output of various edge detection techniques applied in (g) image

4. RESULT

Table 1: SSIM values of various filters

\begin{tabular}{|l|l|l|l|l|}
\hline $\begin{array}{l}\text { Differ } \\
\text { ent } \\
\text { Image } \\
\text { s of } \\
\text { Iris }\end{array}$ & Sobel & Prewitt & LoG & $\begin{array}{l}\text { Min.contructor } \\
\text { of laplacian } \\
\text { edge detector }\end{array}$ \\
\hline $\mathrm{a}$ & $3.49165 \mathrm{e}$ & $3.94381 \mathrm{e}$ & 0.0025941 & 0.0028153 \\
\hline $\mathrm{b}$ & $-2.8772 \mathrm{e}$ & $-1.87133 \mathrm{e}$ & 0.0020141 & 0.0119238 \\
\hline
\end{tabular}




\begin{tabular}{|l|l|l|l|l|}
\hline c & $-2.75792 \mathrm{e}$ & $-1.47375 \mathrm{e}$ & 0.0015134 & 0.0099203 \\
\hline $\mathrm{d}$ & $7.15838 \mathrm{e}$ & $4.39474 \mathrm{e}$ & $-3.37233 \mathrm{e}$ & 0.0049200 \\
\hline $\mathrm{e}$ & $-5.67525 \mathrm{e}$ & $-1.01076 \mathrm{e}$ & -0.0003138 & 0.0064094 \\
\hline f & $-2.4396 \mathrm{e}$ & $-9.77049 \mathrm{e}$ & 0.0029285 & 0.0092613 \\
\hline $\mathrm{g}$ & $-2.81019 \mathrm{e}$ & $-1.83009 \mathrm{e}$ & 0.0007359 & 0.0022296 \\
\hline
\end{tabular}

\section{ACKNOWLEDGMENTS}

The authors are highly grateful and thankful to the Dr. Baljit Singh Khehra(Head Of CSE \& IT Department) of the Baba Banda Singh Bahadur Engineering College, Fatehgarh Sahib and to the college also for making the research complete.

\section{CONCLUSION \& FUTURE SCOPE}

An edge detector is basically a high pass filter that can be applied to extract the edge points in images. The edge detection is the primary step in identifying an image object. This work has compared various edge detecting techniques, Edges of an text image is detected using, Sobel, Prewitt, Laplacian, LOG, minimum constructor with laplacian edge detector and iris image is used for experimentation. The performance of these edge detection methods are analyzed and compared by using the parameter SSIM. It have been observed that that the minimum constructor with laplacian edge detections technique have produced higher accuracy in detection of edges compared with other edge detection algorithms.

\section{FUTURE SCOPE}

In the future scope the results can be compared by using different edge detection techniques like canny edge detection and different parameters can be used like PSNR and MSE.

\section{REFERENCES}

[1] Daouk, C.H., El-Esber, L.A., Kammoun, F.D. and Al Alaoui, M.A. (2002) Iris Recognition. IEEE ISSPIT, Marrakesh, 1.

[2] Tuama, A.S. (2012) Iris Image Segmentation and Recognition. International Journal of Computer Science \& EmergingTechnologies, 3, 60-65.

[3] Shashi Kumar, D.R., Raja, K.B., Chhootaray, R.K. and Pattnaik, S. (2011) PCA Based Iris Recognition Using DWT. International Journal of Computer Technology and Applications, 2,pp. 884-893

[4] Dewangan, A.K. and Siddhiqui, M.A. (2012) Human Identification and Verification Using Iris Recognition by Calculating Hamming Distance. International Journal of Soft Computing and Engineering (IJSCE), 2,pp. 334-338.

[5] Santos, G. and Hoyle, E. (2012) A Fusion Approach to Unconstrained IrisRecognition. Pattern Recognition Letters,

33,pp.984990.http://dx.doi.org/10.1016/j.patrec.2011.08. 017

[6] Daugman, J., "Complete Discrete 2-D Gabor Transforms by Neural Networks for Image Analysis and Compression", IEEE Transactions on Acoustics, Speech, and Signal Processing, Vol. 36, no. 7, July 1988, pp. 1169-1179.
[7] Daugman,J. "How Iris Recognition Works", available at http://www.ncits.org/tc_home//m1htm/docs/m1020044.p df.

[8] Daugman, J., "High Confidence Visual Recognition of Persons by a Test of Statistical Independence,'IEEE transactions on pattern analysis and machine intelligence, vol. 15, no.11, November 1993, pp. 1148-1161.

[9] W. Frei and C. Chen, "Fast Boundary Detection: A Generalization and New Algorithm," IEEE Trans. Computers, vol. C-26, no. 10, pp. 988-998, Oct. 1977.

[10] J. Canny, "A computational approach to edge detection," IEEE Trans. Pattern Analysis and Machine Intelligence, Vol. 8, No. 6, pp. 679-698, Nov. 1986

[11] Raman Maini and Dr. Himanshu Aggarwal "Study and Comparison of various Image Edge Detection Techniques" International Journal of Image Processing (IJIP), Vol3: Issue (1).

[12] S.Lakshmi and Dr.V.Sankaranarayanan "A study of Edge Detection Techniques for Segmentation Computing Approaches" IJCA Special Issue on Imaging and Biomedical Applications" CASCT, 2010. [3] Edge Detection by Trucco,Chapter 4 and Jain ctal.,Chapter5

[13] Zolqemine Othman, habibollahharon, Mohammed Rafi, Abdul kadir, - Comparison of canny and Sobel edge detection in mri images.

[14] M sudarsha*" p ganga Mohan and suryakanth v gangashetty -Optimized edge detection algorithm for face recognition".

[15] Lye Wil Liam, Chekima, A., Liau Chung Fan, and Dargham, J.A., "Iris recognition using selforganizing neural network," IEEE, Student Conference on Research and Developing Systems, Malaysia, pp. 169-172, 2002

[16] Proenca H. and Alexandre L.A., "Iris segmentation methodology for non-cooperative recognition," IEE Proc.Vis.Image Signal Processing, Vol. 153, No. 2, Pp.199-205, 2006.

[17] J. Daugman, "How Iris Recognition Works", Proceedings of 2002 International Conference on Image Processing, Vol. 1, 2002.

[18] R.P. Wildes, Asmuth, J.C. et al., "A System for Automated Iris Recognition", Proc of the Second IEEE Workshop on Applications of Computer Vision, 1994, pp.121 -128

[19] C. Tisse, L. Martin, L. Torres and M. Robert, "Person identification technique using human iris recognition", St Journal of System Research , 2003, Vol. 4, pp. 67-75

[20] W. Boles and B. Boas hash, "A Human Identification Technique Using Images of the Iris and Wavelet Transform," IEEE Trans. on Signal Processing, vol. 46, no. 4, pp.1185-1188, 1998.

[21] R. Wildes, J. Asmuth, G. Green, S. Hsu, R. Kolczynski, J. Matey, S. McBride, "A Machine-vision System for Iris Recognition,” vol. 9, pp.1-8, 1996.

[22] Jain, A., Hong, L., and Pankanti, S. (2000). Biometric identification. Communications of the ACM, 43(2),pp.90-98.

[23] Vatsa, M., Singh, R., and Noore, A. (2008). Improving iris recognition performance using segmentation, quality 
enhancement, match score fusion, andindexing. Systems, Man, and Cybernetics, Part B: Cybernetics, IEEE Transactions on, 38(4),pp.1021-1035.

[24] Chen, W., Er, M. J., and Wu, S. (2006). Illumination compensation and normalization for robust face recognition using discrete cosine transform in logarithm domain. Systems, Man, and Cybernetics, Part B: Cybernetics, IEEE Transactions on, 36(2),pp.458-466

[25] EdurneBarrenechea, HumbertoBustince, Member, IEEE, Bernard De Baets,And Carlos Lopez-Molina, Student Member, IEEE, "Construction Of Interval-Valued Fuzzy Relations With Application To The Generation Of Fuzzy Edge Images", IEEE Transactions On Fuzzy Systems, Vol. 19, No. 5, October 2011, pp. 819-830.

[26] Abdallah A. Alshennawy, and Ayman A. Aly, "Edge Detection Indigital Images Using Fuzzy Logic Technique", World Academy Of Science Engineering And Technology 51, 2009, pp. 178-186.
[27] U.G.Sefercik, O.E.Gulegen, "Edge Detection in geologic formation extraction: Close range and remote sensing Case studies"

[28] H. Voorhees and T. Poggio," Detecting textons and texture boundries in natural images" ICCV 87:250-25, 1987

[29] Pinaki Pratim Acharjya, Ritaban Das and Dibyendu Ghoshal, "Study and Comparison of Different Edge Detectors for Image Segmentation", Global Journal of Computer Science and Technology Graphics \& Vision, Online ISSN: 0975-4172 \& Print ISSN: 0975-4350, Volume 12 Issue 13 Version 1.0 Year 2012

[30] Nisha, Rajesh Mehra and Lalita Sharma "Comparative Analysis of Canny and Prewitt Edge Detection Techniques used in Image Processing”, International Journal of Engineering Trends and Technology (IJETT) -Volume 28 Number 1 - October 2015 